\title{
COMPACT NON-ORIENTABLE SURFACES OF GENUS 4 WITH EXTREMAL METRIC DISCS
}

\begin{abstract}
GOU NAKAMURA
Dedicated to Professor Yoshihiro Mizuta on the occasion of his 60th birthday

Abstract. A compact hyperbolic surface of genus $g$ is said to be extremal if it admits an extremal disc, a disc of the largest radius determined by $g$. We know how many extremal discs are embedded in a non-orientable extremal surface of genus $g=3$ or $g>6$. We show in the present paper that there exist 144 non-orientable extremal surfaces of genus 4 , and find the locations of all extremal discs in those surfaces. As a result, each surface contains at most two extremal discs. Our methods used here are similar to those in the case of $g=3$.
\end{abstract}

\section{INTRODUCTION}

Let $S$ be a compact hyperbolic surface of genus $g$, where $g$ is the number of handles $(\geq 2)$ or that of cross caps $(\geq 3)$ according to whether or not $S$ is orientable. The surface is naturally endowed with a metric induced by the hyperbolic metric of the unit disc $\mathbb{D}$. C. Bavard showed in [1] that the radius of a disc isometrically embedded in $S$ is bounded by the constant $R=R_{g}$ depending only on $g$ :

$$
\cosh R_{g}=\frac{1}{2 \sin \frac{\pi}{6-6 \chi_{g}}}
$$

where $\chi_{g}$ denotes the Euler characteristic; that is, $\chi_{g}=2-2 g$ or $2-g$ if $S$ is orientable or not, respectively. We call $S$ an extremal surface if it contains an extremal disc, a disc of radius $R_{g}$. Our problem is to show how many extremal discs are embedded in an extremal surface. In the case that $S$ is orientable, it was solved for every genus $g>3([2]), g=2([3]$ and [6]), and $g=3([7])$. In the case that $S$ is non-orientable, we know the following for $g>6$ and $g=3$ (4]): an extremal surface of genus $g>6$ contains a unique extremal disc; there exist 11 extremal surfaces of genus 3 , and each of them contains at most two extremal discs. Whereas our methods used here are similar to those in the case of $g=3$, we have to deal with a large number of surfaces for $g=4$.

Received by the editors March 27, 2008.

2000 Mathematics Subject Classification. Primary 30F50; Secondary 30F40.

Key words and phrases. Extremal discs, Klein surfaces.

This work was supported in part by Grant-in-Aid for Young Scientists (B) (No. 20740081).

$$
\begin{array}{r}
\text { (C)2009 American Mathematical Society } \\
\text { Reverts to public domain } 28 \text { years from publication }
\end{array}
$$


Our main result is as follows:

Theorem 1.1. There exist 144 non-orientable extremal surfaces $S_{j}(j=1, \ldots, 144)$ of genus 4, which are derived from the side-pairing patterns $P_{j}$ of the hyperbolic regular 18-gon. They admit at most two extremal discs, and 22 of them admit exactly two extremal discs. $\left(P_{j}\right.$ is listed in the last part of this paper (Figures 3 8), where lines and dotted lines in the figures indicate pairs of sides pasted by the different and the same direction respectively; bullets correspond to the centers of extremal discs.) Tables 1 and 2 show the centers of extremal discs and the full group of automorphisms for each $S_{j}$, where $\pi: \mathbb{D} \rightarrow S_{j}$ denotes the natural projection, $\beta=\pi / 18$, and $R=R_{4}$.

TABLE 1. The list of surfaces of genus 4 admitting two extremal discs.

\begin{tabular}{|l|l|c|}
\hline Surface & Centers of extremal discs & Aut $^{ \pm}$ \\
\hline \hline$S_{1}, S_{89}$ & $\pi(0), \pi\left(\tanh \frac{R}{2}\right)$ & $D_{2} \times \mathbb{Z}_{2}$ \\
\hline$S_{5}, S_{22}, S_{65}$ & $\pi(0), \pi\left(\frac{1}{\sinh R}\right)$ & $\mathbb{Z}_{2} \times \mathbb{Z}_{2}$ \\
\hline$S_{9}, S_{14}, S_{15}, S_{133}, S_{141}$ & $\pi(0), \pi\left(\tanh \frac{R}{2}\right)$ & $\mathbb{Z}_{2} \times \mathbb{Z}_{2}$ \\
\hline$S_{17}, S_{21}, S_{94}$ & $\pi(0), \pi\left(\frac{i}{2 \tanh R \cos \beta}\right)$ & $\mathbb{Z}_{2} \times \mathbb{Z}_{2}$ \\
\hline$S_{53}$ & $\pi(0), \pi\left(\tanh \frac{R}{2}\right)$ & $\mathbb{Z}_{2}$ \\
\hline$S_{69}, S_{78}, S_{135}$ & $\pi(0), \pi\left(\frac{1}{2 \tanh R \cos 2 \beta}\right)$ & $\mathbb{Z}_{2} \times \mathbb{Z}_{2}$ \\
\hline$S_{76}, S_{142}$ & $\pi(0), \pi\left(\frac{2 \sin 2 \beta}{\tanh R} i\right)$ & $\mathbb{Z}_{2}$ \\
\hline$S_{77}, S_{81}, S_{139}$ & $\pi(0), \pi\left(\frac{2 \sin 2 \beta}{\tanh R} i\right)$ & $\mathbb{Z}_{2} \times \mathbb{Z}_{2}$ \\
\hline
\end{tabular}

TABLE 2. The list of surfaces of genus 4 admitting a unique extremal disc.

\begin{tabular}{|l|c|}
\hline Surface & Aut $^{ \pm}$ \\
\hline \hline$S_{138}, S_{144}$ & $D_{6}$ \\
\hline$S_{4}, S_{68}$ & $D_{3}$ \\
\hline$S_{73}, S_{87}, S_{121}, S_{127}, S_{137}, S_{143}$ & $\mathbb{Z}_{2} \times \mathbb{Z}_{2}$ \\
\hline$S_{3}, S_{6}, S_{8}, S_{11}, S_{12}, S_{13}, S_{16}, S_{18}, S_{28}, S_{29}, S_{40}, S_{41}, S_{42}$, & \\
$S_{43}, S_{55}, S_{56}, S_{63}, S_{64}, S_{66}, S_{67}, S_{70}, S_{74}, S_{82}, S_{83}, S_{84}$, & $\mathbb{Z}_{2}$ \\
$S_{85}, S_{86}, S_{88}, S_{90}, S_{91}, S_{92}, S_{93}, S_{100}, S_{101}, S_{114}, S_{115}$, & \\
$S_{119}, S_{123}, S_{128}, S_{131}, S_{136}, S_{140}$ & $\{1\}$ \\
\hline The others & \\
\hline
\end{tabular}

In order to obtain this theorem we follow the same line of the proof for the case of genus 3 (cf. 44).

\section{Side-PAIRING PATTERnS OF THE 18-GON}

Since a fundamental region for a non-orientable extremal surface of genus $g \geq 3$ is a hyperbolic regular $(6 g-6)$-gon, we shall find every side-pairing pattern for 
the regular 18-gon which yields a non-orientable surface of genus 4. Identifying the equivalent sides, we have a trivalent graph with 9 edges and 6 vertices on the surface. It is easily shown that there are 17 such trivalent graphs (Figure 1). Note that $M, N, O, P$, and $Q$ appear in [5].

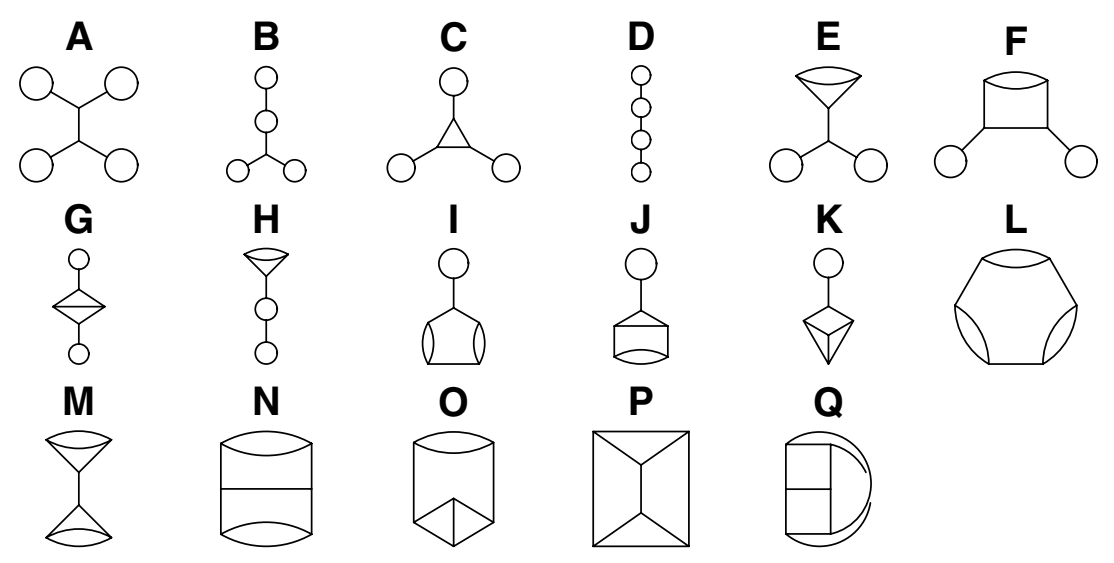

FIGURE 1. Trivalent graphs with 6 vertices

Considering the closed walks on these graphs by computer, we obtain all sidepairing patterns (cf. [4, §5.1]).

Theorem 2.1. A regular 18-gon admits 144 side-pairing patterns $P_{1}, \ldots, P_{144}$ which yield non-orientable surfaces of genus 4 (Figures 3 8). Table 3 shows the correspondence between the trivalent graphs and the side-pairing patterns, e.g., $P_{1}$ is obtained from $A$.

TABLE 3. The trivalent graphs and the side-pairing patterns

$\left.\begin{array}{|l|l|}\hline A & P_{1} \\
B & P_{2} \\
C & P_{3}, P_{4} \\
D & P_{5}, P_{6} \\
E & P_{7}, P_{8}, P_{9} \\
F & P_{10}, \ldots, P_{16} \\
\hline\end{array}\right]$\begin{tabular}{ll|l|l|}
$G$ & $P_{17}, \ldots, P_{22}$ \\
$H$ & $P_{23}, \ldots, P_{26}$ \\
$I$ & $P_{27}, \ldots, P_{33}$ \\
$J$ & $P_{34}, \ldots, P_{47}$ \\
$K$ & $P_{48}, \ldots, P_{62}$ \\
$L$ & $P_{63}, \ldots, P_{68}$ \\
\hline
\end{tabular}

\section{Extremal Discs For the 144 SURfaces}

In this section we shall sketch the proof of Theorem 1.1. Without loss of generality we may assume that the center of a hyperbolic regular 18-gon is the origin and that the vertices $v_{n}$ satisfy $\arg v_{n}=(2 n-1) \pi / 18(n=1, \ldots, 18)$ in the unit disc $\mathbb{D}$. The origin therefore projects to the center of an extremal disc. We denote by $C_{n}$ the side between $v_{n}$ and $v_{n+1}$, and by $w_{n}$ the middle point of $C_{n}$, where subscripts are regarded as modulo 18.

The following lemma is shown by using the same process as [4, Lemma 5.3]. 
Lemma 3.1. Let $S$ be a non-orientable extremal surface of genus 4 and $\pi: \mathbb{D} \rightarrow S$ the natural projection. If $p$ is the center of an extremal disc of $S$, then the hyperbolic distances between two points in the set $\pi^{-1}(p)$ are listed such as $d_{1}=2 R \approx 3.438$, $d_{2} \approx 4.746, d_{3} \approx 5.496$, and so on.

Note that $d_{2}=2 l+s$, where $l$ denotes the hyperbolic distance between the origin and a vertex $v_{n}$, and $s$ the hyperbolic length of a side $C_{n}$ (Figure 2).

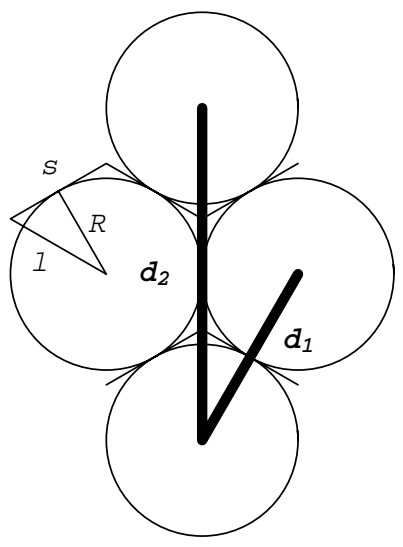

FiguRE $2 . d_{1}$ and $d_{2}$

Lemma 3.2. Let $K_{n}(n=1, \ldots, 18)$ be the pentagon of which vertices are $w_{n-1}$, $v_{n}, v_{n+1}, w_{n+1}$, and the origin. Let $t_{n}=t_{n, m}$ be the side-pairing mapping from $C_{n}$ onto the other side $C_{m}$. If $z$ is a point of $K_{n}$ and is projected to the center of an extremal disc, then $d\left(z, t_{n}(z)\right)$, the hyperbolic distance between $z$ and $t_{n}(z)$, is equal to $2 R$.

Proof. Whereas our proof is similar to that of [4, Lemma 5.4], we shall describe it without numerical values. We know that $d\left(z, t_{n}(z)\right)$ is less than or equal to the maximum of distances between a vertex of $K_{n}$ and its image by $t_{n}$, and that $d\left(0, t_{n}(0)\right)=2 R, d\left(v, t_{n}(v)\right) \leq 2 l\left(v=v_{n-1}, v_{n}\right)$, and $d\left(w, t_{n}(w)\right) \leq R+l+s / 2$ $\left(w=w_{n-1}, w_{n}\right)$. It is then obvious from the triangle inequality that $2 R<2 l<$ $R+l+s / 2$. Therefore we have $d\left(z, t_{n}(z)\right) \leq R+l+s / 2$. Since $R+l+s / 2<2 l+s=d_{2}$ holds by the triangle inequality again, we have $d\left(z, t_{n}(z)\right)=d_{1}=2 R$.

Suppose that $z \in K_{n}$ is projected to the center of an extremal disc. By the equation $d\left(z, t_{n, m}(z)\right)=2 R, z$ must be on the curves $L_{n, m}$ or $M_{n, m}$ (or curves $L_{n, m}^{\prime}$ or $M_{n, m}^{\prime}$ ) if $t_{n, m}$ is orientation preserving (or reversing) (see 4, Lemma 5.5]):

$$
\begin{aligned}
& L_{n}=L_{n, m}:\left|z-\frac{\tanh R e^{i(n+m) \beta}}{2 \cos (n-m) \beta}\right|=\frac{\tanh R}{2|\cos (n-m) \beta|}(n-m \not \equiv 9 \quad(\bmod 18)), \\
& M_{n}=M_{n, m}: z=\operatorname{coth} R e^{2 i n \beta}-t e^{i(n+m+9) \beta}(t \in \mathbb{R}), \\
& L_{n}^{\prime}=L_{n, m}^{\prime}:\left|z-\frac{\operatorname{coth} R e^{i(n+m) \beta}}{2 \cos (n-m) \beta}\right|=\frac{\operatorname{coth} R}{2|\cos (n-m) \beta|}(n-m \neq \equiv 9 \quad(\bmod 18)), \\
& M_{n}^{\prime}=M_{n, m}^{\prime}: z=\tanh R e^{2 i n \beta}-t e^{i(n+m+9) \beta}(t \in \mathbb{R}) .
\end{aligned}
$$



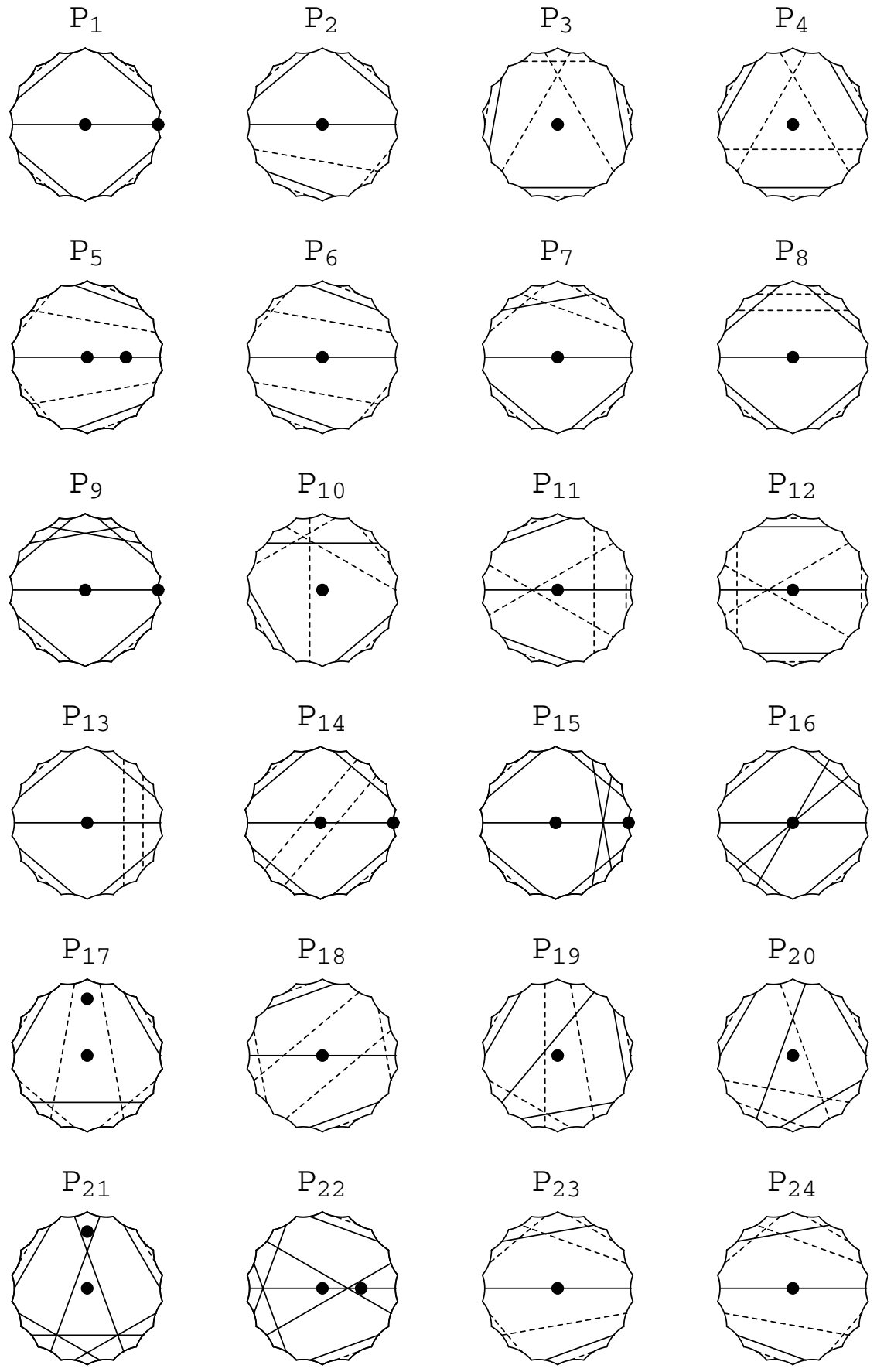

FiguRE 3. Side-pairing patterns and the centers of extremal discs 

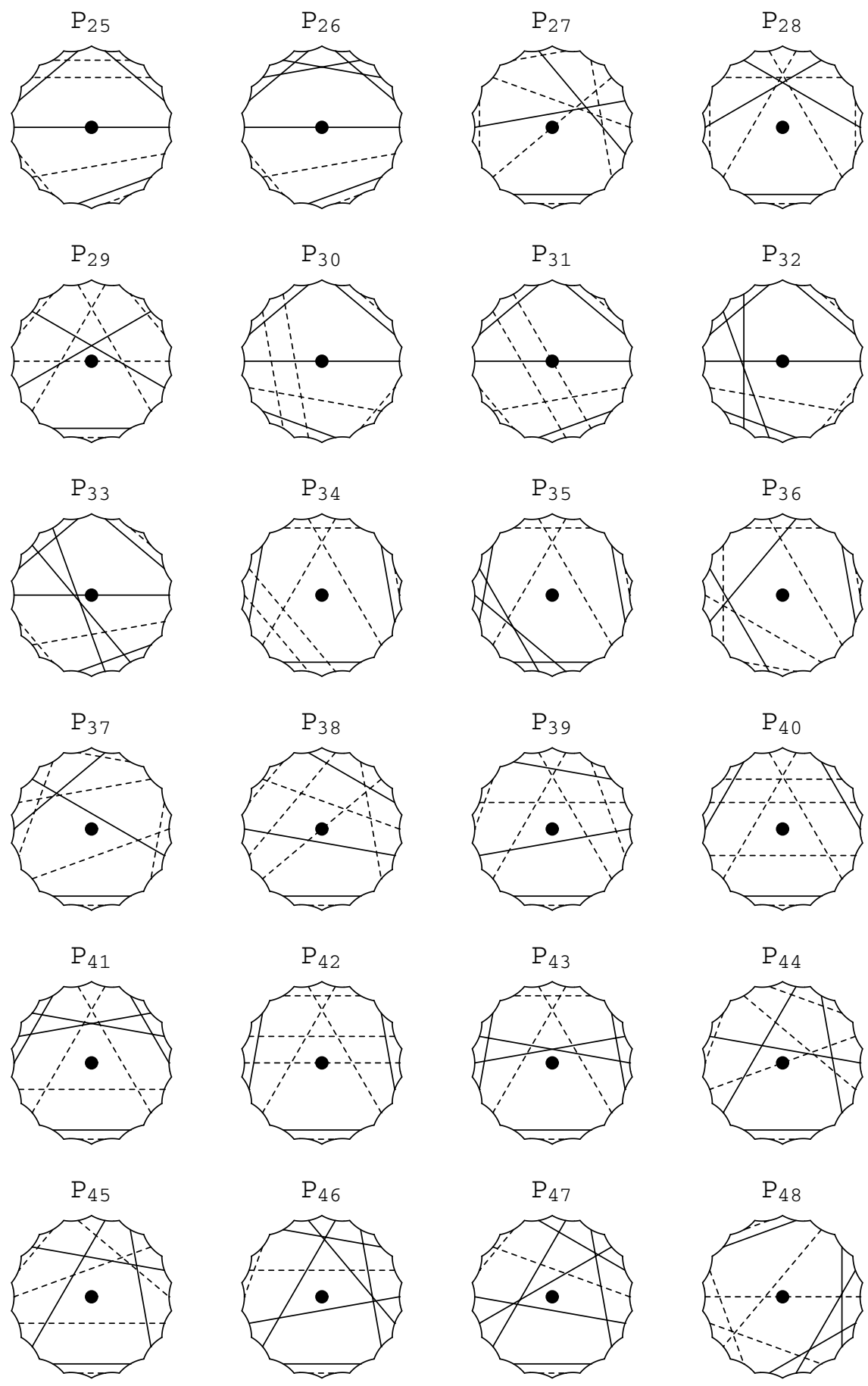

FiguRE 4. Side-pairing patterns and the centers of extremal discs 

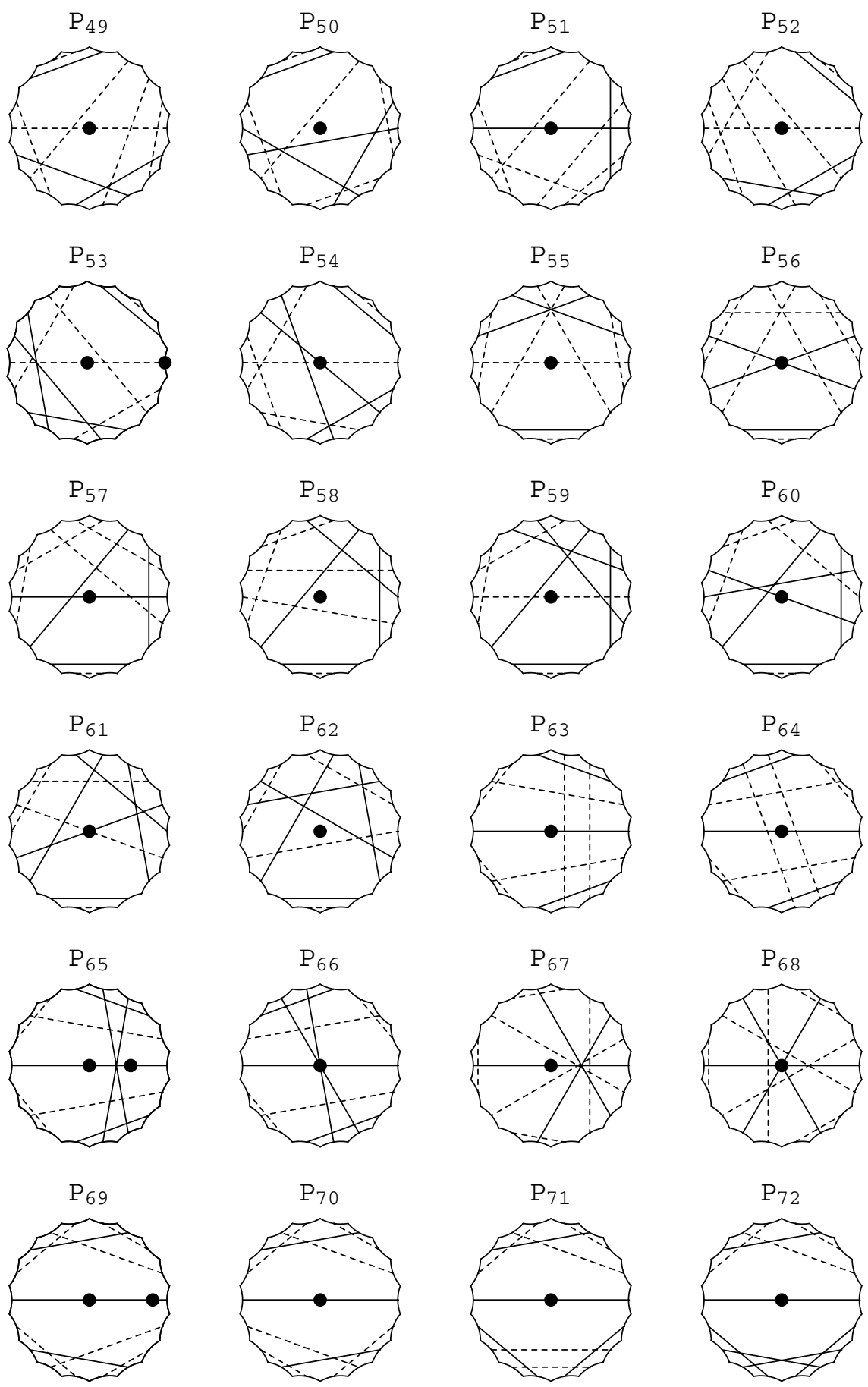

FiguRE 5. Side-pairing patterns and the centers of extremal discs 

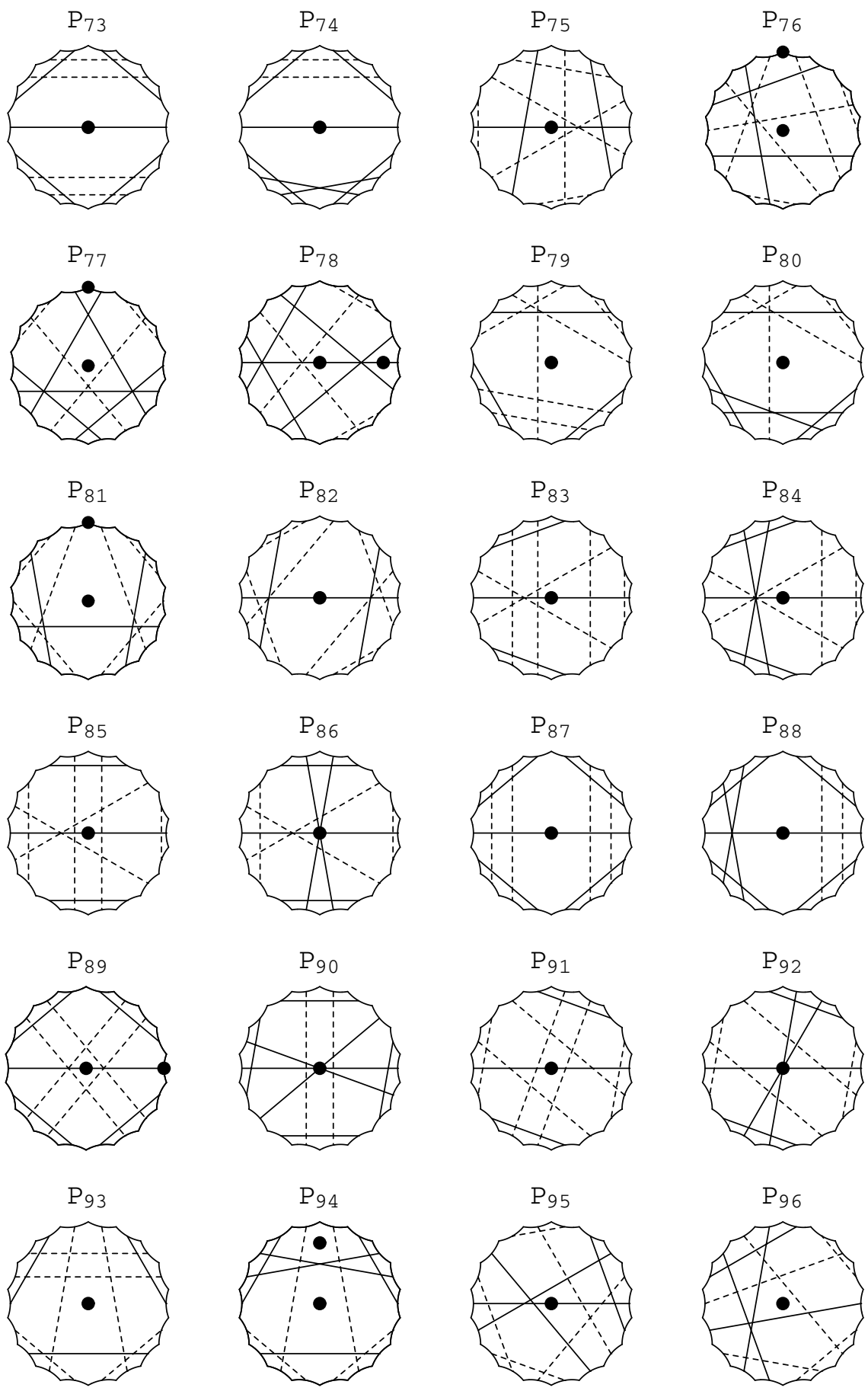

FiguRE 6. Side-pairing patterns and the centers of extremal discs 

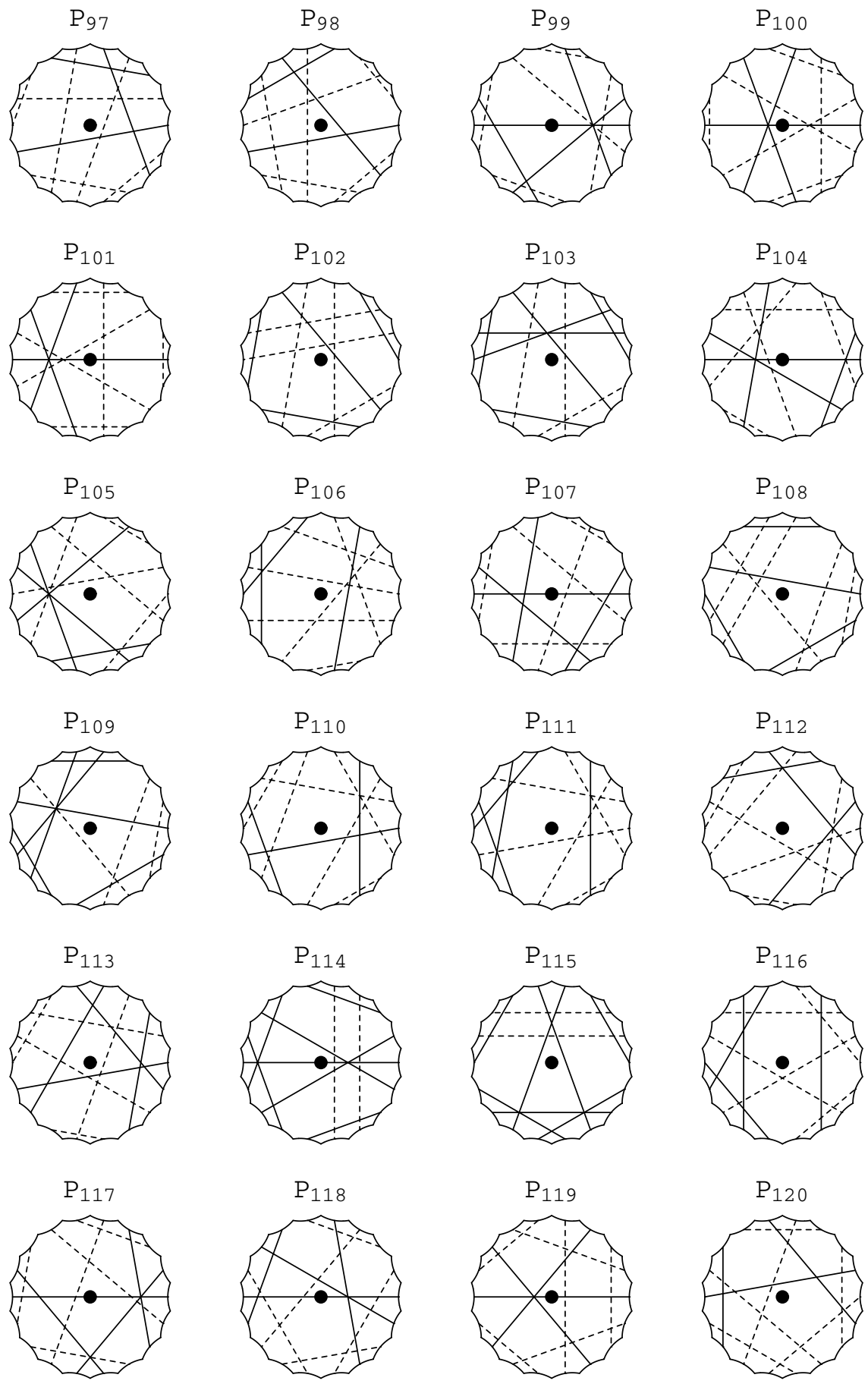

FiguRE 7. Side-pairing patterns and the centers of extremal discs 

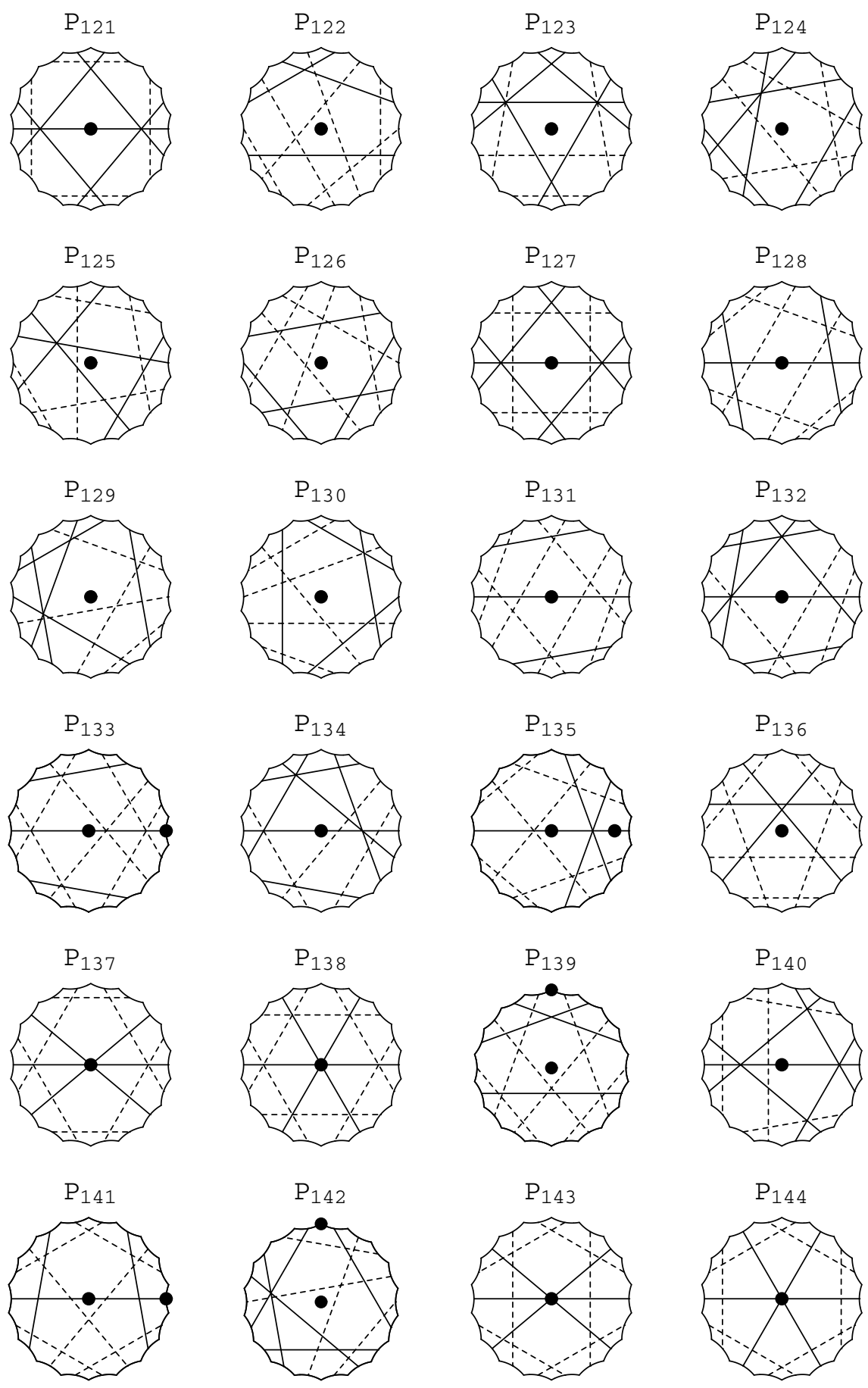

FiguRE 8. Side-pairing patterns and the centers of extremal discs 
In order to accomplish our purpose, we shall refer to a process to find the center of an extremal disc.

(1) According to a side-pairing pattern $P_{j}$, draw $L_{n}, M_{n}$ (or $L_{n}^{\prime}, M_{n}^{\prime}$ ) on $K_{n}$ for every $n$;

(2) find intersections of these curves;

(3) select every point $\zeta$ in the intersections such that $d\left(\zeta, t_{k}(\zeta)\right)$ is in the list of Lemma 3.1 for every side-pairing mapping $t_{k}$ of $P_{j}$.

Applying this process to $P_{j}(j=1, \ldots, 144)$, we see that 22 side-pairing patterns admit two points and that the others admit a unique point. We can show that the two points (one is the origin) arising from the 22 patterns are transitive by some isometry $f \in \mathrm{Aut}^{ \pm}(\mathbb{D})$ compatible with the side-pairing mappings. Since the origin projects to the center of an extremal disc, so does the other point.

Example 3.3. As an example we shall consider $P_{1}$. Through the process above we get two points $\zeta=\tanh (R / 2)$ and the origin. Put $f(z)=(\zeta-z) /(1-\bar{\zeta} z)$, then we can verify that $f$ is compatible with the side-pairing mappings of $P_{1}$ :

$$
\begin{array}{ll}
f \alpha_{1,4} f^{-1}=\alpha_{14,17}, & f \gamma_{2,3} f^{-1}=\gamma_{15,16}, \\
f \alpha_{5,8} f^{-1}=\alpha_{9,0} \alpha_{17,14}, & f \gamma_{6,7} f^{-1}=\alpha_{9,0} \gamma_{12,11} \alpha_{17,14}, \\
f \alpha_{9,18} f^{-1}=\alpha_{18,9}, & f \alpha_{10,13} f^{-1}=\alpha_{4,1} \alpha_{0,9}, \\
f \gamma_{11,12} f^{-1}=\alpha_{4,1} \gamma_{7,6} \alpha_{0,9}, & f \alpha_{14,17} f^{-1}=\alpha_{1,4}, \\
f \gamma_{15,16} f^{-1}=\gamma_{2,3}, &
\end{array}
$$

where $\alpha_{n, m}$ and $\gamma_{n, m}$ denote the orientation preserving and reversing side-pairing mapping from $C_{n}$ to $C_{m}$, respectively. Consequently, the projection $\pi(\zeta)$ is the center of an extremal disc.

The surfaces derived from $P_{j}(j=1, \ldots, 144)$ are not isomorphic to each other (see [4, Remark 5.9]). Furthermore it is not difficult to determine the full group of automorphisms by the fact that the centers of extremal discs are fixed or interchanged by automorphisms (cf. 4, Corollary 5.10]).

For example, Aut ${ }^{ \pm}\left(S_{1}\right)=D_{2} \times \mathbb{Z}_{2}=\left\langle\sigma, J_{0}\right\rangle \times\langle T\rangle\left(=\mathbb{Z}_{2} \times \mathbb{Z}_{2} \times \mathbb{Z}_{2}\right)$, where $\sigma, J_{0}$, and $T$ are automorphisms induced by $z \mapsto-z, z \mapsto \bar{z}$, and $z \mapsto f(z)$, respectively.

\section{ACKNOWLEDGEMENTS}

The author is very grateful to Ernesto Girondo for his helpful comments and suggestions.

\section{REFERENCES}

1. C. Bavard, Disques extrémaux et surfaces modulaires, Ann. de la Fac. des Sciences de Toulouse V (1996), no. 2, 191-202. MR1413853 (97i:30059)

2. E. Girondo and G. González-Diez, On extremal discs inside compact hyperbolic surfaces, C. R. Acad. Sci. Paris, t. 329, Série I (1999), 57-60. MR1703263 (2000f:53036)

3. E. Girondo and G. González-Diez, Genus two extremal surfaces: extremal discs, isometries and Weierstrass points, Israel J. Math. 132 (2002), 221-238. MR1952622 (2003k:57020)

4. E. Girondo and G. Nakamura, Compact non-orientable hyperbolic surfaces with an extremal metric disc, Conform. Geom. Dyn. 11 (2007), 29-43. MR2295996 (2007k:30087)

5. T. Jørgensen and M. Näätänen, Surfaces of genus 2: generic fundamental polygons, Quart. J. Math. Oxford (2), 33 (1982), 451-461. MR679814 (84c:51029) 
6. G. Nakamura, The number of extremal disks embedded in compact Riemann surfaces of genus two, Sci. Math. Japon 56 (2002), no. 3, 481-492. MR1937911(2003i:30065)

7. G. Nakamura, Extremal disks and extremal surfaces of genus three, Kodai Math. J. 28 (2005), no. 1, 111-130. MR2122195(2005j:30055)

Science Division, Center for General Education, Aichi Institute of Technology, YAKUSA-CHO, TOYOTA 470-0392, JAPAN

E-mail address: gou@aitech.ac.jp 